\title{
Téoros
}

Revue de recherche en tourisme

\section{Le tour du bout du monde. Un siècle de tourisme en Gaspésie}

\section{Marie-Claude Guérette et Pierre Hétu}

Volume 14, numéro 2, été 1995

Le tourisme : toute une histoire!

URI : https://id.erudit.org/iderudit/1075093ar

DOI : https://doi.org/10.7202/1075093ar

Aller au sommaire du numéro

Éditeur(s)

Université du Québec à Montréal

ISSN

0712-8657 (imprimé)

1923-2705 (numérique)

Découvrir la revue

Citer cet article

Guérette, M.-C. \& Hétu, P. (1995). Le tour du bout du monde. Un siècle de tourisme en Gaspésie. Téoros, 14(2), 8-11. https://doi.org/10.7202/1075093ar d'utilisation que vous pouvez consulter en ligne.

https://apropos.erudit.org/fr/usagers/politique-dutilisation/ 


\section{Le tour du bout du monde Un siècle de tourisme en Gaspésie}

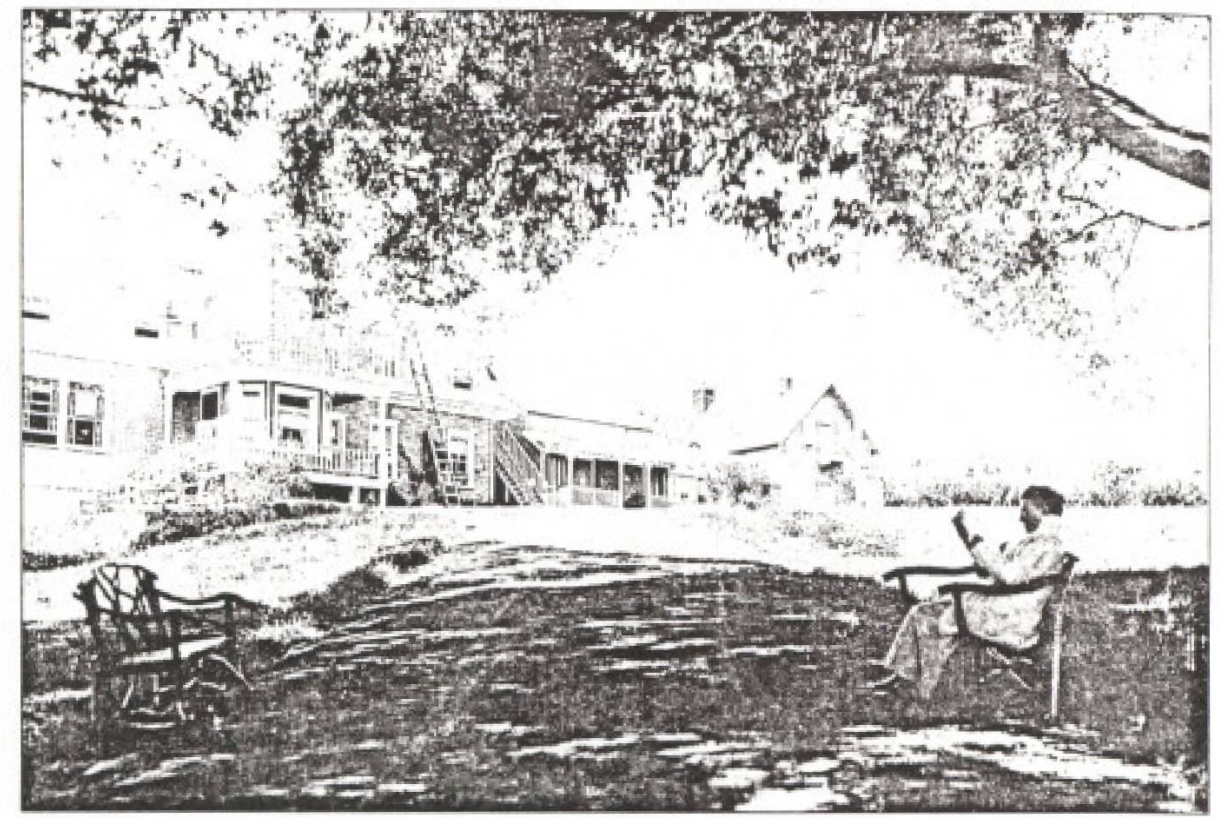

L'Hôtel Baker de Gaspé au début du siècle. Photographie noir et blane provenant de la collecrion G.G. Mercier (P92), Centre d'Archives de la Gaspésie, Musée de la Gaspésie.

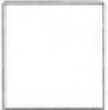

Parmi les circuits touristiques offerts au Québec, il en est un qui se distingue par sa force évocatrice et par la présence combinée de la mer, de la forêt et de la montagne: Le tour de la Gaspésie! En effet, qui, de sa jeunesse, ne se souvient pas de son premier périple familial en terregaspésienne? Leur vie durant, d'aucuns considèrent ce circuit comme une sorte de pèlerinage. Depuis longtemps déjà, la Gaspésie est une terre de prédilection pour des générations de voyageurs. Fréquentée par des estivants fortunés, des scientifiques et desartistes au siècle dernier, la péninsule s'est progressivement ouverte à une clientèle plus diversifiée au cours des années 1930, pour devenir la destination populaire que l'on connait aujourd'hui. Cette trajectoire touristigue remarquable ne s'est jamais démentie. A la suite des Nord-Américains, les Européens ont succombé au charme gaspésien. Remontons dans le temps pour découvrir les origines et l'évolution de cette passion pour le Finistère québécois.
Madame Marie-Claude Guerette est recherchiste et assistante à la réalisation et Monsieur Pierre Hétuest rédacteur cher Cinémanima inc. Québec.

\section{La villégiature}

La notion de vacances ne date pas d'hier. Toutefois, longtemps cette pratique a-telle été le privilège des riches qui s'adonnaient à la villégiature. Du reste, le terme villégiature tire son origine de l'italien villegiatura, qui signifie *aller à la campagne». Ainsi, l'été venu, les familles aristocratiqueset bourgeoises des citéss'offraient des séjours en province où elles occupaient de somptueuses villas construites sur des sites remarquables; la durée de leur séjour variait de quelques semaines à quelques mois. Le reste de l'année, ces résidences secondaires ne servaient pas; le plus souvent, on les placardait. Les premiers vacanciers à visiter la péninsule gaspésienne sont donc d'influentes familles anglosaxonnes, canadiennes ou américaines, qui fuient l'activité trépidante et là pollution des villes le temps d'une saison.

La Gaspésie du XIX ${ }^{t}$ siècle est alors un refuge idéal car elle conserve toujours ce caractère pittoresque si convoité par les villégiateurs en quête d'originalité: la population locale poursuit ses activités tradi- 
tionnelles comme la pêche, l'agriculture, la coupe du bois et la construction navale. Cet immense territoire offre de nombreux paysages encore vierges. D'un point de vue social, les deux univers different tellement que leurs contacts se limitent à l'essentiel. Forts de leur supériorité matérielle et de leur culture urbaine, les citadins observent les paysans et les pêcheurs avec un mélange de sympathie et de condescendance. A leur décharge, soulignons que les péninsulaires sont alors très peu scolarisés. Ce retard en matière d'éducation est directement relié au système de troc déloyal que leur imposent les marchands jersiais. Ce régime les a longtemps maintenus dans un état de pauvreté ignominieux. Il n'y a pas besoin d'instruction pour eux, écrivait monsieur Philippe Robin à ses commis; s'ils étaient instruits en seraient-ils plus habiles à la pêche.s.(1) Seuls quelques diaristes comme Arthur Buies, l'abbé Ferland, Faucher de Saint-Maurice, Effie Molt-Bignell ou le docteur Von Iffland tentent de dépasser les apparences.

Dès la fin du XVIII siècle, Guy Carleton (lord Dorchester), gouverneur du Canada, vient séjourner dans la baie des Chaleurs quelques étés. A sa suite, des membres de l'aristocratie anglaise ainsi que des bourgeois canadiens-anglais et américains commencent à fréquenter la région. Il faut toutefois attendre les années 1850 pour que le mouvement s'amplifie et que la Gaspésie acquiert sa réputation de centre de villégiature. De somptueuses villas et de grands hôtels commencent alors à apparaìtre sur le pourtour de la péninsule. Ces touristes bien nantis fréquentent principalement le sud-est de la péninsule soit la baie de Gaspé, la Percésie et la Baie-desChaleurs. L'hớtel Baker à Gaspé, le Bleu Blanc Rouge à Percé, l'hôtel Annettà New Carlisle, le Chăteau Blanc à Bonaventure ou le White House à Carleton ne sont que quelques-uns des joyaux qui ont fait de la Gaspésie une destination distinctive et recherchée.

En partance de Montréal ou de Québec, ces vacanciers de la belle société s'embarquent sur des bateaux de croisière pour la Gaspésie. Les vapeurs de la Québec \& Gulf Port Steamships, de la Gaspé Steamship Line ou encore de la Clark Steamships font escale à différents points de la côte, pour laisser descendre les voyageurs parvenus à destination. Pendant longtemps, peu de villages sont équipés d'un quai; les bateaux moullent aux larges des côtes et de petites embarcations transhordent les passagers. Le dépliant promotionnel de la Gaspé Steamship Line de 1912 est assez éloquent sur la nature de la croisière.

Un voyage par eau de sept cent milles sur le Saint-Lawent jusqu'au Golfe et à La Baie des Chaleurs procurera au citadin énervé wne dizaine de jours de jouisance saine et salubre. On ne saurait concevoir promenade plus pittoresque et plus restawrante.

\section{$[\ldots]$}

Aux ports d'arrit des chaloupes abordent le bateau pour faire l'écbange des passagers et apporter des provisions pour la table d'bôte, y compris des difices inconnus aux babitants des pays d'en baut, notamment du saumon frais toutà-fait autbentique, de la truite et de la morue frais-sortic de la mer. [sic]

L'arrivée du train marque toutefois le véritable essor touristique gaspésien. En 1898, les ramifications du chemin de fer s'étendent jusqu’à New Carlisle, en empruntant la vallée de la Matapédia. Mais ce n'eşt qu'en 1911 que le train atteint Gaspé. A l'ouverture de chacune des nouvelles stations, le réseau hôtelier se consolide pour accommoder la nouvelle clientèle.

Les activités récréatives sont simples. On prend des bains de mer, on hume l'air salin, on contemple le paysage pittoresque et, bien sür, on taquine le saumon. Les hôtels les plus chic offrent à leurs clients la possibilité de jouer au tennis, au croquet ou encore d'aller en mer à la voile. Au milieu du XTX' siècle, des géologues, des botanistes, des naturalistes commencent à mener des missions en Gaspésie pour évaluer et cataloguer les richesses naturelles; plus tard, au tournant du siècle, des artistes se joignent aux savants et aux wanciers pour s'inspirer des splendeurs du panorama. Parmi les figures les plus illustres, on remarque celles du frèreMarie-Victorin, de William Logan, de M. L. Fernald, de H. W. McGerrigles, de Frederick James, de Marc-Aurêle Fortin, d'Alberto Tommi et du pape du surréalisme en personne, André Breton.

\section{Les clubs de chasse et de pêche}

Parallèlement à la villégiature, se développent en Gaspésie les clubs privés de chasse et de pêche. A partir des années 1870 , des Américains ou des Canadiens anglais puissants s'accaparent peu à peu des meilleures rivières du territoire gaspésien en louant les rives du gouvernement ou encore en achetant les bordures des cours d'eau aux habitants. Le mouvement prend une telle ampleur, qu'en 1884, le gouvernement promulgue la loi dite des Trois Chaines qui lui réserve les ubordures de rivières sur une largeur de 198 pieds ( 60 mètres) [une chaîne représente 66 pieds ( 20 mètres)] sur l'ensemble des terres non concédées de la Couronne avec tous les droits de pêche existants $w^{(0)}$, ce qui lui permet d'affermer ses rivières et, bien sür, d'en tirer de substantiels profits. Enfin, en 1885, l'Etat vote une loi favorisant la naissance de clubs de pêche. Ainsi, naîtront de prestigieux clubs privés tels Restigouche Salmon Club et Matamajaw Salmon Club. Les financiers influents et les politiciens s'y rencontrent avecleurs relations et profitent del'expertise d'un guide pour descendre les rivières; on leur indiquealors lesmeilleures fosses pour la pêche. Leurs prises sont apprêtées sur place par un personnel qui se charge aussi de les mettre sur la glace et de les expédier aux quatre coins de l'Amérique.

\section{L'explosion touristique}

Jusqu'au début du siècle donc, les rives de la Gaspésie ne sont accessibles qu'à une minorité d'estivants privilégiés. Il faut attendre le train, mais surtout le développement du réseau routier pour voir se dessiner le profil actuel de la Gaspésie touristique. En effet, avant la construction du boulevard Perron ${ }^{(3)}$ en 1927-1929, aucune route ne ceinture la péninsule dans sa totalité. Mais avec la construction de cette woie, toute la Gaspésie s'ouvre au grand public.

Des événementsmarquants ponctuent cette constante évolution. En 1934, plus de 20000 personnes assistent aux célébrations du 400 'e anniversaire de l'arrivée de Jacques Cartier au Canada quiont lieuà Gaspé. Cet événement majeur annonce le début d'une nouvelle forme de tourisme, le tourisme nomade.

\section{[...] quelque 30000 personnes se sont donné rendez-vous sur les rives de cette baie, los 24,25 et 26 août}


1934, [... J ces fêtes ont été l'ocasion du plus grand rassemblement de visiteurs et de persomnalitér jamais vu en Gaspésie. Des convois ferroviaires spécianux de Montréal et Québec ont débarqué des milliers de viriteurs sur le quai de la gare de Gaspé. Des navires de guerre de France, de Grande-Bretagne, des Etats-Unis et du Canada trônaient dans la baie alors que sur les routes de la péninsule d'interminables convois de vébicules automobiles convergeaient vers Gappé.

\section{[... Une fois les bôtels et les pensions} occupés, les visiteurs étaient dirigés vers les 3000 chambres recensées dans les maisons entre Rivière-auRenard et Percé alors que le college et le couvent étaient transformés en dortoir. [... . Le Canadien National, de son côté, avait mis à la disposition de sa dientéle quelgue 58 wagonslits rangés sur une voie de garage de un mille de long.(")

De quoi faire rêver les organisateurs des fêtes du $450^{\circ}$ qui allait se dérouler à Québec en 1984 !

Cette explosion phénoménale, momentanément interrompue par la Deuxième Guerre mondiale, se confirme après 1945. Le phénomène ressortit d'une nouvelle conjoncture: d'une part, l'automobile devient plus accessible pour la cla sse moyenne et, d'autre part, la diminution des heures de travail et la généralisation des vacances estivales favorisent l'explosion touristique. Dès lors, les Gaspésiens doivent s'adapter à une clientèle plus diversifiée qui séjourne moins longtemps au même endroit.

On instaure un nouveau type d'hébergement pour accueillir ce flot de visiteurs. On assiste d'abord à l'érection de nom= breuses cabines et d'hôtels à prix modique, puis, dans les années 1950 , les motels font leur apparition et les terrains de camping croissent en nombre. Les activités récréatwes se diversifient elles aussi pour offrir toute une gamme de nouvelles expériences telles que le deltaplane, les excursions en canot, les randonnées à cheval, la plongée sous-marine ou l'observation de mammiferes marins.

\section{Le gouvernement et le développement touristique}

Avec les richesses naturelles, le tourisme est l'un des éléments moteurs de l'économie gaspésienne. Dès le début des années 1930, le gouvernement entend développer ce volet névralgique d'un territoire oublié, sans structure industrielle organisée à part lespêcheries. Un premier effort est consenti dans la promotion de cette région qui devient une synecdoque du tourisme québécois. En 1928, 500000 cartes postales représentant la Gaspésie sont distribuées. Puis, en 1929, dans le sillon de l'inauguration du boulevard Perron, on publie un guide touristique La Gaspésie: Histoire, Légender, Ressources, Beautés. Les hyperboles et le lyrisme rivalisent pour décrire *ce qui est aujourd'hui reconnu comme le plus grand centre de villégiature du continent américain w que l'on n'hésite pas à comparer à l'Irlande:

Ce que fut l'Irlande dans les siedles passés, y lit-on, la Gaspésie lest aujourd bui. Le progrès moderne ne la pas dépouillée de son charme et de ses attraits naturels. Bien que l'bomme ait, depuis plus de trois cents ans, enva hi ses vastes solitudes et parcouru toutes ses rives, nulle part il n'a laisse de manifestations tangibles de son désir inné de conquérir la nature.

\section{$[\ldots]$}

L'accès de cette région prodigiense, où la monitagne et la mert s'unissent dans un même paysage grandiose, de cette sterre promises du chasseur et du pêcheur, a été rendlu facile aux atutomobilistes gräce à La construction, par le ministere de la Voirie de la province de Québer, de la route désignée sous le nom de aboulevard Perrons, (5)

Viendront ensuite de nombreux articles promotionnels dans différentes revues populaires tant au Canada anglais qu'aux Etats-Unis. Mais l'intervention du gouvernement ne se limite pas à la publicité et à la promotion; elle vise aussi l'implantation d'une infrastructure d'accueil et la mise en place d'équipements récréo-touristiques pour les estivants.

Plusieurs projets ont étéstuucturés dans les années 1930 et 1940 telle la créstion du
Parc de la Gaspésie en 1937 et son ouverture à la population en 1949. Toutefois, l'implication en force des gouvernements fédéral et provincial, dans de nouvelles infrastructures touristiques, correspond à la mise sur pied du Bureau d'aménagement de l'Est du Québec (BAEQ) en 1963 et, surtout, à la signature de l'Entente CanadaQuébec de 1968. À l'origine, le BAEQ devait servir à évaluer le potentiel socioéconomique de la région et à formuler des recommandations pour que l'économie gaspésienne entre enfin dans le marché nord-américain. Le tourismes'est retrouvé en tête de liste des agents de promotion économique et de nombreux projets favorisant son essor ont été réalisés, parmi lesquels figurent la création du parc national Forillon et la mise en valeur de nombreux sites historiques et culturels. En outre, plusieurs infrastructures d'accueil, telles que des haltes routières ou des terrains de camping, ont poussé comme des champignons. Ces équipements récréo-touristiquesont permisà la Gaspésiede maintenir, et même d'élargir, ses capacités d'accueil et de récréation et d'en faire l'une des destinations les plus recherchées au Québec.

\section{Quelques ombres au tableau}

Àl'instar de tous les ruccess story, l'épopée du tourisme gaspésien a connu ses épisodes moins glorieux. En dépit d'un apport économique incontestable, la présence d'étrangers investis de tous les droits génère quelques frustrations qui se traduisent parfois par des confrontations entre les habitants et leur tout-puissants visiteurs.

L'affaire Coffin, qui a défrayé la chronique judiciaire à partir de 1953, constitue peutêtre la manifestation la plus tragique de ce malaise. Ce malheureux fait divers n'est pas étranger à l'histoire du tourisme en Gaspésie. L'histoire commence par la disparition, puis par la découverte des corps detroischasseurs américainsen 1953. Parmi l'équipe qui aide la Police provinciale à mener les recherches figure un prospecteur anglophone de Gaspé qui se trouve à être la dernière personne à avoir vu les trois chasseurs vivants. L'enquête policière conclutà à un acte criminel et le solliciteur général recherche un meuruier.

Le prospecteur, du nom de Wilbert Coffin, est arrêté et traduit en justice. L'année suivante il est reconnu coupable à partir de preuves circonstancielles. Il sera pendu en 
février 1956. Le procès de Coffin comporte plusieurs vices de forme qui seraicnt inacceptables de nos jours. Cependant, les défenseurs de Coffin prétendent que les politiciens de l'époque avaient à tout prix besoin d'un coupable, de peur que les pêcheurs et les chasseurs étrangers désertent notre territoire, privant l'Etat d'entrées importantes de capitaux. Au nombre des plus virulents opposants aux conclusions de ce procès, le journaliste Jacques Hébert clame, dans un pamphlet incendiaire, que: «L'analyse même sommaire de l'enquête judiciaire, le compte-rendu du procès ultra-rapide qui s'est déroulé à Percé, les nouvelles preuves versées aux dossiers depuis le procès, démontrent que Wilbert Coffin a été pendu malgré le doute manifeste qui persistait et persiste encore. $\$^{(6)}$

Partout danslemonde, lessites touristiques donnent lieu à des excès qui risquent de compromettre leur valeur même. La Gaspésie n'a pas été exempte de tels abus. Mais il s'est toujours trouvé quelques voix plus lucides pour les dénoncer. Déjà dans les années 1930, l'évéque de Gaspé, Monseigneur Ross, combattait l'anglicisation galopante qui s'emparaitalors de l'affichage commercial, «Il incite les Gaspésiens à embellir leurs propriétés et à démontrer de l'originalité dans la fabrication de souvenirs et dans l'apprêt des mets. Il insiste particulièrementsur la fabrication desenseignes, s'attaquant aux "Inns", aux "Camping Grounds", aux "Home Spun", aux "Hot Dogs" et aux "Fresh Fish" lance de tous les instants stimpose lorsque l'invasion estivale s'annonce.

À partir des années 1960, le mercantilisme donne aussi lieu à certains excès, notamment dansle village de Percé, qui est de loin le plus fréquenté de la péninsule. Victime de sa popularité, Percé s'apparente à un endroit où $\propto[. .$.$] l'odeur des frites remplace$ de plus en plus celle de la morue et on peut en parler comme de l'OLd Orchard Beacb du Québec. [...] La cuisine est plusaméricaine que gaspésienne et l'ardisanat se fait volonters japonais ${ }^{(8)}$ Le déclin de l'industrie touristique de la fin des années 1970 permet au Gaspésiens de rajuster le tir et de cesser la destruction inconsidéré du patrimoine gaspésien. Depuis, on s'applique à offrir un produit davantage en harmonie avec la couleur locale.

Aujourd hui, le tourisme gaspésien a acquis une grande maturité et il demeure un vaisseau amiral de certe industrie pour le
Québec. Il a su développer de nouveaux marchés et attirer une clientěle internationale. Cette trajectoire enviable résulte évidemment d'un environnement à nul autre pareil, mais aussi à l'esprit de concertation qui anime les Gaspésiens. Qu'il s'agisse du Finistère ou de l'Irlande, la Gaspésie supporte la comparaison haut la main. $\boldsymbol{f}$

\section{BIBLIOGRAPHIE}

BELANGER, Jules, DESJARDINS, Marc et Jean-Yues FRENETTE (avec la collaboration de Fierre Danse. reau), Histoire de la Gaspésie, Montréal, Borfal Express/institut de recherche sur ls cullure, 1981. BUAEAU PRIONINCIAL DU TOURISME, La Gasptsie: Histoire. Legendes. Remources, Beautes. Québec, ministère de la Voirie, 1930

FAUCHER DE SAINTT-MAURICE, Henri-Edmond, De tribord ta babord, Montréal, L'Auroce, 1975. Pre. sentation de Jacques Ferronet notes de Ghislaine Beaulieu.

FAUCHER DE SAINT-MAUAICE, Henti-Edmond, La Gaspasie : promenade dans le golfe Saint-Laurent, Montréal, Librairie Saint Joseph, 1886.

HEBERT, Jacques, Coffin btait innocent, Montréal, Les Editions de l"Homme, 1958

LEMIEUX, Paul, Cest artive chez nous.... Tourismechasse-peche-loisir. L'histoire d'un ministere dana I'Eat du Oubec, Ouébec, ministère du Laisir, de la Chasse et de la Péche, 1986.

MOLT-BIGNEU, Effie, Lavie quotidienne en Gaspesie su dabut du sibcle, Sainte-Anne-des-Monts. Editions de la SHAM, 1983.

RASTOUL, Pierre et Alan ROSS, La Gaspésie, de Grosen-Roche a Gaspe. Itináraire eulturel. Québec, Libraine Beauchemin/Edteur officiel du Québec, 1978 (Collection des guides pratiques). La Gasptaie (Revue d'histoire de la Gaspesie).

\section{NOTES}

(1) L'abbe Ferland cité par Faucher de Saint-Maurice dans De tribord babord presentation de Jacques Ferron, notes de Ghislaine Beaulieul. Montréal, L'Aurore, 1975, p. 207.

(2) Paul LEMIEUX, Cest arrive chez nous..., Tou risme-chasso-pache-loisir. L'histoir d'un mi. nistere dans r'Est du Oubbec, Québec, mins. tetre du Loisir, de la Chasse et de la Peche, 1966. p. 35 .

(3) Le boulevard a efte nomme enl'honneur de JosephLeonide Perron, ministre de la Voirie sous le gouvernement libéral de Louis-Alexandre Taschereau (1920-1936)

(4) Paul LEMIEUX, op. cit. p. 163.

(5) Bureau provincial du tourisme, La Gasphaie: Histoirt, Légendes, Ressources, Beautba, Quebec. ministère de la Voirie, 1930, p. 10-13

(6) Colfin ttaht innoctent, Montreal, Les éditions de rHomme, 1958, p. 186.

(7) Jules BELLANGER, Marc DESJARDINS, Jean-Yves FRENETTE tavec la collaboration de Pierre Dansereaul, Histoire de la Gaspessie, Montréal. Boréal Express/institut de recherche sur ta culture, 1981, p. 616

(9) lbid. D. 620 